\title{
Nonlinear dynamical response of interacting bosons to synthetic electric field
}

\author{
Arko Roy $\odot,{ }^{1,2}$ Soumya Bera, ${ }^{3}$ and Kush Saha ${ }^{4,5}$ \\ ${ }^{1}$ INO-CNR BEC Center and Dipartimento di Fisica, Universitá di Trento, 38123 Trento, Italy \\ ${ }^{2}$ Max-Planck-Institut für Physik komplexer Systeme, Nöthnitzer Straße 38, 01187 Dresden, Germany \\ ${ }^{3}$ Department of Physics, Indian Institute of Technology Bombay, Mumbai 400076, India \\ ${ }^{4}$ School of Physical Sciences, National Institute of Science Education and Research, Jatni, Odisha 752050, India \\ ${ }^{5}$ Homi Bhabha National Institute, Training School Complex, Anushakti Nagar, Mumbai 400094, India
}

(Received 4 April 2020; accepted 8 October 2020; published 26 October 2020)

\begin{abstract}
We theoretically study the nonlinear response of interacting neutral bosonic gas in a synthetically driven one-dimensional optical lattice. In particular, we examine the bosonic analog of electronic higher harmonic generation in a strong time-dependent synthetic vector potential manifesting itself as the synthetic electric field. We show that the vector potential can generate reasonably high harmonics in the insulating regime, while the superfluid regime exhibits only a few harmonics. In the insulating regime, the number of harmonics increases with the increase in the strength of the vector potential. This originates primarily due to the field-driven resonant and nonresonant excitations in the neutral Mott state and their recombination with the ground state. If the repulsive interaction between two atoms $(U)$ is close to the strength of the gauge potential $\left(A_{0}\right)$, the resonant quasiparticle-quasihole pairs on nearest-neighbor sites, namely, dipole states, are found to a play a dominant role in generating higher harmonics. However, in the strong-field limit $A_{0} \gg U$, the nonresonant states where quasiparticle-quasihole pairs are not on nearest-neighbor sites give rise to higher harmonics.
\end{abstract}

DOI: 10.1103/PhysRevResearch.2.043133

\section{INTRODUCTION}

The interplay between intense laser field and matter continues to be a field of extensive research both theoretically and experimentally as it allows us to decode microscopic mechanisms of several physical systems such as photonic, gaseous, solid-state, and quantum spin systems [1-4]. The nonperturbative nature of the matter-light interaction makes the field even more promising due to its potential for exhibiting unprecedented and rich physics. For example, the generation of higher harmonics in gaseous systems [5-9], leading to plateaus in the energy distribution of emitted light, has stimulated research for several decades and has now become a key candidate for attosecond science [10,11]. Recently, the higher harmonic generation (HHG) has been experimentally observed and theoretically studied in solid-state systems [12-22] and semimetals [23]. While gas-phase HHG is limited to complex experimental setups and millijoule-class laser pumps, the solid-state HHG turns out to be easily achievable and nondestructive. Thus it emerges as a potential platform for ultrafast and short-wavelength coherent light sources [12]. A great volume of work suggested that the HHG in solidstate system can be used to probe the electronic properties of a wide range of materials [24,25]. It has been also shown that engineered solid-state structure [26] can be used as a

Published by the American Physical Society under the terms of the Creative Commons Attribution 4.0 International license. Further distribution of this work must maintain attribution to the author(s) and the published article's title, journal citation, and DOI. Open access publication funded by the Max Planck Society. possible candidate for producing stable extreme-ultraviolet (XUV) wave forms [27].

While most of the studies on HHG in gas and solid-state systems either assume weak interaction or are based on the single-particle picture, only a few recent studies have addressed HHG in strongly correlated electronic systems such as Mott insulators [28-32]. Using the fermionic Hubbard model,

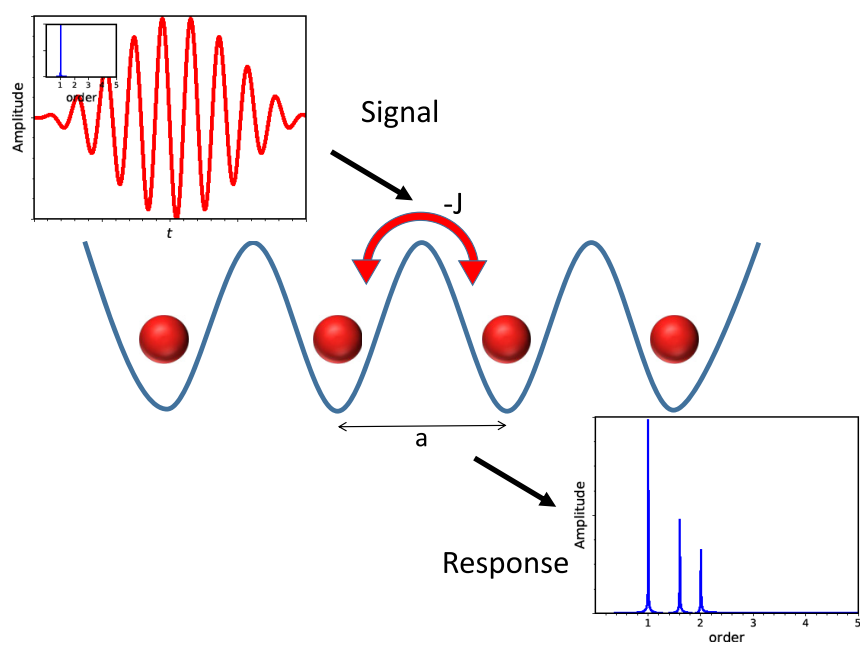

FIG. 1. Schematic diagram of light-atom interaction in a onedimensional optical lattice setup. The synthetic 10 -cycle $\sin ^{2}$ pulse as the time-dependent gauge potential is used with a frequency $\omega / J=30$. The optical response of the system under the pulse is found to exhibit higher harmonics of the input $\omega$. Note that the coupling between neutral atom and light is synthetic in nature as also discussed in the main text. 
it has been shown that the HHG can be used to resolve ultrafast many-body dynamics in a Mott insulator [28,29]. In the strong field, the mechanism that leads to HHG in Mott insulators turns out to be a recombination of field-excited doublon-holon pairs with the ground states. In contrast, in the low-field limit, the itinerant doublon-holon excitations play the role in generating higher harmonics. Given HHG in interacting fermionic Mott insulators, it is natural to ask if analogous higher harmonics can also be obtained in a bosonic Mott insulator of interacting neutral bosons loaded in an optical lattice under a synthetic electric field. If yes, what is the underlying mechanism for the generation of such higher harmonics in the bosonic Mott insulators? Since the typical notion of doublon-holon excitations in fermionic Mott insulators cannot be extended to bosonic systems, this poses a pertinent question that needs to be addressed. Bosons in optical lattices are known to exhibit a class of excitations depending on the strength of the applied electric field [33]. It is also thus important to discern how various types of excitations contribute to HHG.

To address the above questions, we consider the onedimensional Bose-Hubbard model and theoretically study the bosonic analog of electronic HHG in different regimes of atom-atom interactions giving rise to the superfluid and Mott phases. We find that the dynamics of atoms in the superfluid regime is transparent to the synthetic vector potential. In contrast, the Mott-insulating phase exhibits strikingly different optical responses. By varying the strength of the potential, we find the optical transitions involve an increasing order of harmonics in the Mott-insulating regime. The superfluid regime remains unaffected as we increase the strength of the synthetic gauge potential. We show that the generation of higher harmonics in the Mott-insulating regime can be attributed to the formation of resonant dipole states - a pair of quasiparticle and keyhole on nearest-neighbor sites when the potential strength is comparable with the interaction $U$. On the contrary, in the strong-field limit, the HHG is attributed to the nonresonant states where the quasiparticle-quasihole pair resides on different sites.

Concerning experimental adaptability, the first challenge is to achieve a perfect Mott insulator at finite temperature in the context of the present theoretical study. However, this has been overcome by reducing the thermal entropy of the atoms in a novel and efficient way recently as outlined in Ref. [34], leading toward the realization of a strongly correlated Mott-insulating state which is essential for creating HHG. The particle-hole excitations which are essential for $\mathrm{HHG}$, as discussed in this work, can be created either by the synthetic vector potential in the presence of additional Raman lasers, or tilting the lattice with an additional gradient field [35]. Then the subsequent dynamics is expected to be measured in the time-of-flight experiments as illustrated in Refs. [36,37]. We note that recently the response of the interacting ultracold atomic rubidium sample due to the application of the femtosecond laser pulse through the resonant and nonresonant channels has been experimentally studied in Ref. [38]. Although this cold-atom setup is driven by electrons rather than bosons themselves, the identification of the specific channels forms the basis of our understanding behind the production of HHG as will be elucidated in this paper.
The rest of the paper is organized as follows. In Sec. II, we discuss the model and formalism in the presence of a synthetic electric field. In particular, we discuss the one-dimensional Bose-Hubbard model in the presence of a 10 -cycle $\sin ^{2}$ pulse followed by a discussion on the numerical methods and coupling of the electric field with the neutral atoms. This is followed by Sec. III, where we present the HHG spectrum for both Mott and superfluid regimes for different strength of the gauge potential, and explain the possible reason for such HHG. We furthermore show the evolution of fidelity for different atom-atom interaction. Finally, we conclude with a discussion on the possible future directions and experimental implications in Sec. IV.

\section{MODEL AND METHODS}

\section{A. Bose-Hubbard Hamiltonian}

We start with a gas of bosonic atoms at zero temperature trapped in an one-dimensional (1D) optical lattice potential. The statics and dynamics of this bosonic system can well be described by the single (lowest) band Bose-Hubbard (BH) Hamiltonian to emulate real condensed matter systems

$$
\hat{H}=-\sum_{\left\langle l l^{\prime}\right\rangle} J \hat{a}_{l}^{\dagger} \hat{a}_{l^{\prime}}+\frac{U}{2} \sum_{l} \hat{n}_{l}\left(\hat{n}_{l}-1\right)-\mu \sum_{l} n_{l},
$$

where $\left\langle l l^{\prime}\right\rangle$ refers to nearest-neighbors (NNs) $l$ and $l^{\prime}, J$ is the hopping strength between two NN sites, $U$ is the interaction, $\mu$ is the chemical potential, which sets the particle number $N$ in the system, and $\hat{a}_{l}^{\dagger}\left(\hat{a}_{l}\right)$ are the bosonic creation (annihilation) operators with $\hat{n}_{l}=\hat{a}_{l}^{\dagger} \hat{a}_{l}$ as the occupation number in the $l$ th lattice site. The model assumes that any excitation associated with the interaction $U$ is smaller than the separation energy to the first excited band of the deep optical lattice $[39,40]$. Depending on the relative values of $U / J$, this model supports two distinct phases. For $U / J \gg 1$, the system exhibits a gapped insulating phase, namely a Mott insulator (MI) with commensurate integer fillings and vanishing order parameter. The Mott gap is determined by the energy difference between a single-particle excitation band (energy involving adding an extra particle in the Mott phase) and a single-hole excitation band (energy involving removing a particle in the Mott phase). Note that these particle-hole excitation bands are the genesis of higher harmonic orders as will be evident shortly. In contrast, $U / J \ll 1$ leads to the gapless superfluid (SF) phase with nonvanishing compressibility $[35,39,41,42]$. Specifically, the presence of two gapless particle-hole excitation energy bands at $\mathbf{k}=0$ among the four constitutes the relevant superfluid physics [43]. For the present work, we consider the average particle number per site to be 1 in the Mott phase.

\section{B. Synthetic electric field}

As opposed to the HHG in gases and solid materials where electric field $E(t)$ naturally couples to the electrons via timevarying vector potential $A(t)$, the analogous HHG in bosonic neutral atoms arises due to coupling between the $A(t)$ and neutral atoms in a synthetic manner. Specifically, $A(t)$ can be regulated in an experiment by detuning of two Raman lasers which affect the mechanical momentum of the particles. 

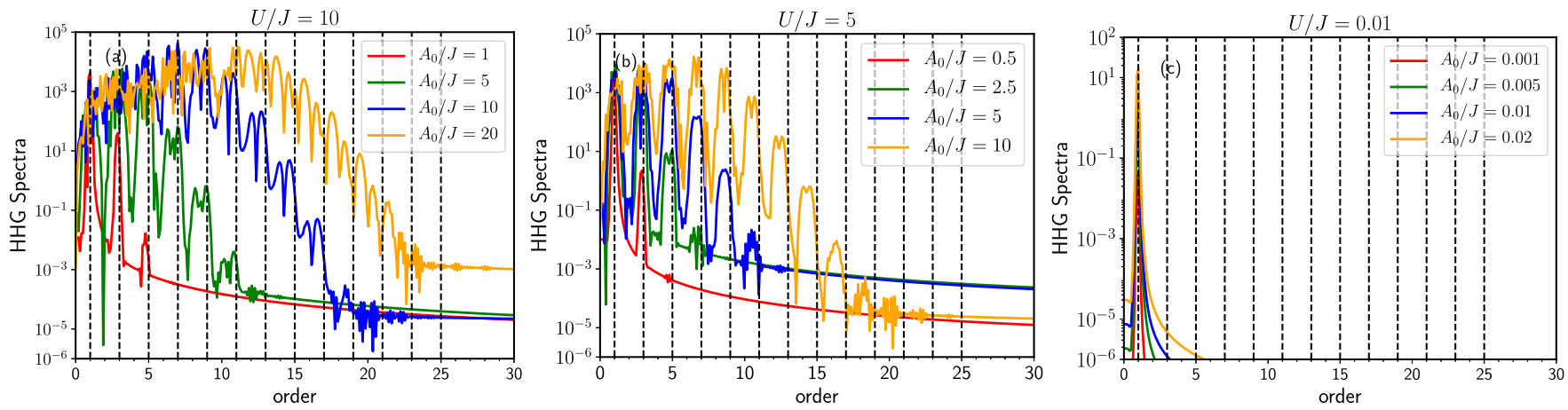

FIG. 2. Higher harmonic spectrum both in the Mott (a), intermediate (b), and superfluid (c) regime for different strength of the electric field. Evidently, in the Mott and intermediate regime, the harmonic order increases with the increase in electric field strength, whereas in the superfluid regime a single peak appears at the driving frequency. The black dashed lines indicate the odd harmonics.

In particular, the multiple internal states of the neutral atom are coupled with slight off-resonant Raman lasers which in turn lead to dressed atoms. These dressed atoms behave like charged particles with an effective charge $q^{*}$ and they move with a finite velocity [36]. This forms the basis of coupling between the neutral atoms and synthetic vector potential. Note that so far the experiments have demonstrated mostly static fields. However, the gauge fields can have externally imposed time dependence giving rise to effective time-dependent electric fields. This can be brought about periodically changing the external magnetic field, which in turn induces time dependence on the Zeeman shift of the atomic spin states. This would change the detuning periodically. There have been several proposals in generating dynamical gauge fields with ultracold atoms; however, experimental limitations have hindered progress $[36,44]$. Considering this experimental fact and for the sake of studying HHG, we use an $n$-cycle $\sin ^{2}$ time-varying vector potential of the form of a pulse, $A(t)=$ $A_{0} \sin ^{2}(\omega t / 2 n) \sin (\omega t)$ with $\omega$ being the frequency of oscillation, where $E(t)=-\partial_{t} A(t)$. The strength of the vector potential $A_{0} \sin ^{2}(\omega t / 2 n)$ smoothly varies with $t$ and the maximum value is attained at the half cycle of the pulse. The $A(t)$ minimally couples to the system via the hopping term as $J e^{i \Phi(t)}$, where $\Phi(t)=q^{*} A(t) a$, with $a$ as the lattice constant. Figure 1 illustrates the schematic of light-atom coupling in a 1D optical lattice setup, which is synthetic in nature.

We point out that the variation of $A(t)$ which leads to the change in momentum with time, in turn, imparts a force on the dressed atoms and constitutes the effective particle current as discussed in Sec. III A. It could be also interpreted as radiation of a neutral system. Also, we note that the $A(t)$ can couple the particle-hole excitations giving rise to various resonant and nonresonant excitations (located within the gap between the lowest and first excited states) depending on the ratio between $U$ and $A_{0}$, where $A_{0}$ is taken in units of energy [33]. This will be discussed in Sec. III B.

\section{Numerical methods}

To study the dynamics, we solve the time-dependent Schrödinger equation $\hat{H}(t) \psi(t)=i \hbar \frac{\partial \psi(t)}{\partial t}$ numerically. The ground state of the interacting Hamiltonian [Eq. (1)] at $t=0$ is computed for a lattice site of length $L=7$ and total number of atoms $N=7$, using exact diagonalization. It is then evolved under time-dependent Hamiltonian $\hat{H}(t)$ using the RungeKutta algorithm choosing an optimum temporal step size which renders the dynamics convergent (see Appendix A). For a detailed review on the use of numerical methods to study HHG, the reader may refer to Ref. [45]. With the evolved wave function, various quantities are computed to investigate the response of the interacting many-body system to the synthetic pulse. It may be mentioned here that with the increase in the system size, the dimension of the Hilbert space increases exponentially, and thus computing the dynamics becomes computationally expensive. We have thus refrained here from providing results for large system sizes; however, we have checked that the mechanism for HHG remains unchanged with the increase in system size, which is discussed in Appendix B.

\section{RESULTS AND DISCUSSION}

\section{A. HHG spectrum}

To investigate the effect of light field, we first evaluate the current operator defined as

$$
\mathcal{J}(t)=-i a q^{*} J \sum_{i=1}^{L}\left(e^{-i \Phi(t)} b_{i}^{\dagger} b_{j}-\text { H.c. }\right) .
$$

The HHG spectrum is obtained from the dipole acceleration $F(t)=d \mathcal{J} / d t$ in frequency space. Note that the nonlinear current generated due to synthetic driving in this present study does not emit photons, in contrast to the real materials. Figure 2 shows HHG spectra in deep Mottinsulating, intermediate, and deep superfluid regimes for different strength of the gauge potential $A_{0}$. Evidently, there is a single dominant peak in the superfluid regime and the location of the peak does not change with the change in the strength of the potential. This is attributed to the lowest quasienergy band current without any higher quasienergy curve crossings, and is usually typical of any single-band Hamiltonian under periodic driving [11]. As mentioned before, since the superfluid phase is gapless, there is only one band and the application of synthetic electric field leads to typical Bloch-type oscillation within this band. In contrast, the Mott-insulating phase, which is characterized by a gapped excitation spectrum, exhibits optical transitions between the quasienergy levels brought about by the coupling of 


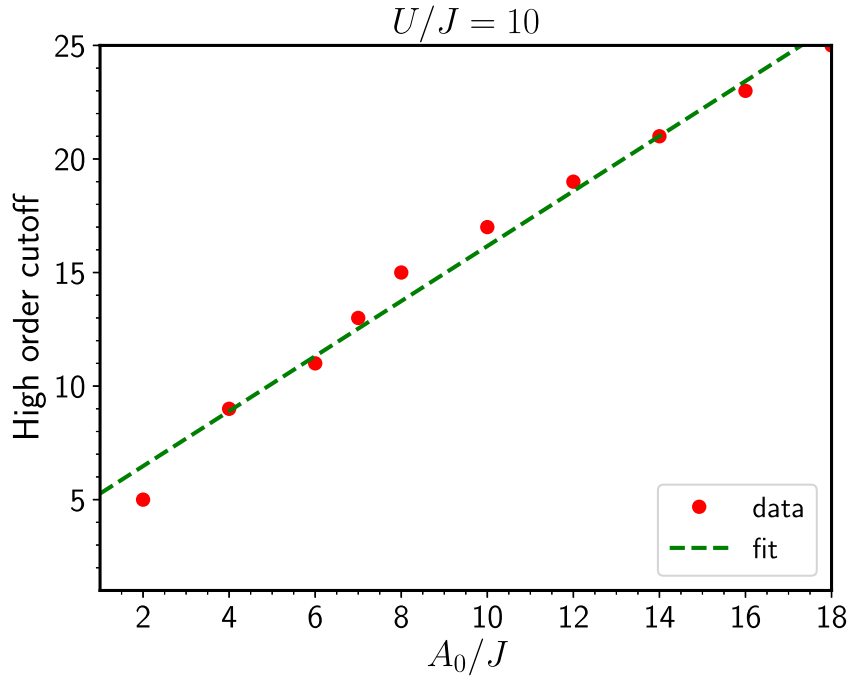

FIG. 3. Dependence of the high-order harmonic spectrum on the strength of the gauge potential for $U / J=10$ revealing a linear cutoff law.

particle-hole excitations. This is further associated with higher harmonics of the synthetic driving frequency. When $U / J=5$, that is, in the intermediate regime, higher harmonics are also generated with the application of the synthetic light pulse as evident from Fig. 2(b). However, the value of the highest order harmonic for any $A_{0} / J$ is less than what is obtained in the deep insulating regime. Furthermore, it is to be noted here that unlike the fermionic system studied in Ref. [28], as we increase the synthetic field strength $A_{0}$, the order of harmonics increases monotonically. The cutoff scaling law with the strength of the vector potential $A_{0}$ turns out to be linear as shown in Fig. 3, corroborating the typical scaling obtained in gases and solids [3,12].

For low-order harmonics, the peaks appear exactly at odd-integer multiples; however, they deviate slightly for highorder harmonics. As we increase the strength of the gauge field, the deviation starts to increase. This may be attributed to the unitary dynamics of the model that we consider. The absence of the bath-induced dephasing terms in the current Hamiltonian leads to the offset of the odd-harmonic spectrum for the higher orders [28,46,47]. Cold atoms in optical potentials provide extremely clean conditions and do not suffer from dissipation induced by the coupling to phonons. However, dissipation can be introduced to get clear HHG peaks through a non-Hermitian Hamiltonian, which is beyond the scope of our present study. Furthermore, in some cases, the prominent splitting of the peaks may be attributed to the current due to the coupled quasienergy levels similar to the two-band Bloch oscillation in a static field, where the dynamics of the two-band systems is characterized by two timescales as explained in Ref. [48].

The reason for the HHG in the bosonic system may be associated with the recombination of resonant and nonresonant excitations with the ground state in the presence of a synthetic vector potential. These excitations do not have analogs to the real systems, and the mechanism of HHG in real solids and gases is different. It is worth pointing out here that the energy scales for our current setup should not be strictly compared

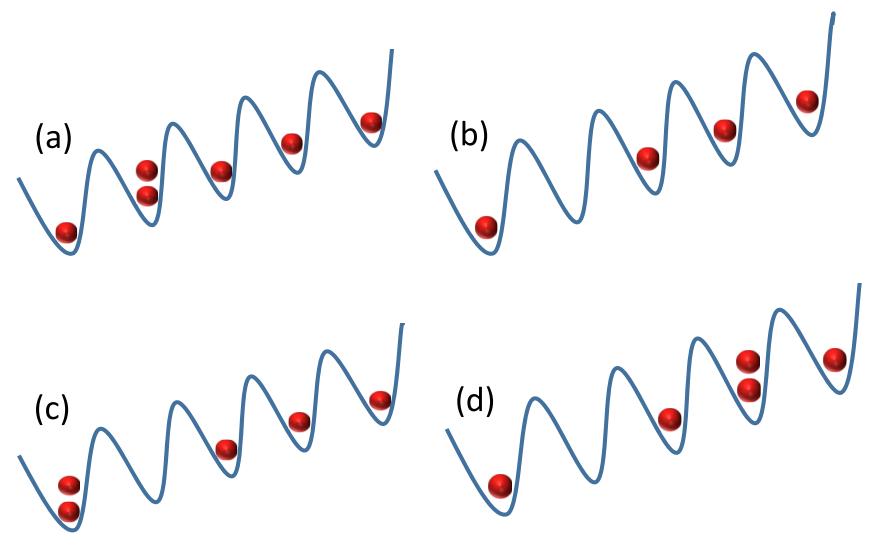

FIG. 4. Schematic representation of deformed Mott insulators in a field-driven tilted optical lattice. (a) A quasiparticle state at site 2, (b) a quasihole state at site 2, (c) a resonant state with a pair of quasiparticle-quasihole states on nearest-neighbor sites (this state is usually called the dipole state), and (d) a nonresonant state with a quasiparticle-quasihole pair not on nearest-neighbor sites.

to that of real solid-state systems or gases. Whether it be cold atom or real materials, since the related physics should be scale-independent, we use normalized dimensionless units in all the relevant plots and discuss the mechanism. Thus, to understand the role of these excitations in HHG in a coldatom setup, we first briefly review typical excitations in the Mott phase of an optical lattice in the presence of a static electric field.

\section{B. Excitations in the Mott-insulating phase}

A static electric field $E$ introduces the Stark term $H^{\prime}=$ $E \sum_{i} i a_{i}^{\dagger} a_{i}$ in Eq. (1), which is equivalent to tilt or a linear potential gradient in the optical lattice in the coordinate space. We also note that the addition of $H^{\prime}$ is the same as modifying hopping $J$ by the Peierls phase involving electric field as discussed before. They are related by a simple gauge transformation as shown in Ref. [49]. It is convenient to understand the excitation spectrum of the system and subsequent tunneling between the sites in terms of tilting of the optical lattice. In the Mott limit, i.e., $J \ll U$, the typical "quasiparticle" and "quasihole" excitations can be created by adding a single particle on a site or removing one particle from a site (see Fig. 4), respectively. Such quasiparticle or quasihole states over the Mott states turn out to be localized even in the presence of any finite gauge potential [33]. Consequently, these states do not extend across the whole system to produce significant changes in the initial state. Thus such excitations of a deformed Mott state with net finite charge cannot take part in generating higher harmonics. On the other hand, a Mott state with zero net charge, usually called the neutral Mott state, produces various families of excitations in the presence of a synthetic electric field. The excitations of such Mott state are possible only if $E \sim U$, where $E$ is measured in units of energy. In this limit, a quasiparticle-quasihole pair is formed on nearest-neighbor sites and they may tunnel resonantly into the nearest site. These states are called dipole states [33] and differ in energy from the Mott state by $\sim E-U$ when $J=0$. Thus at $U=E$, states become degenerate and an infinitesimal 

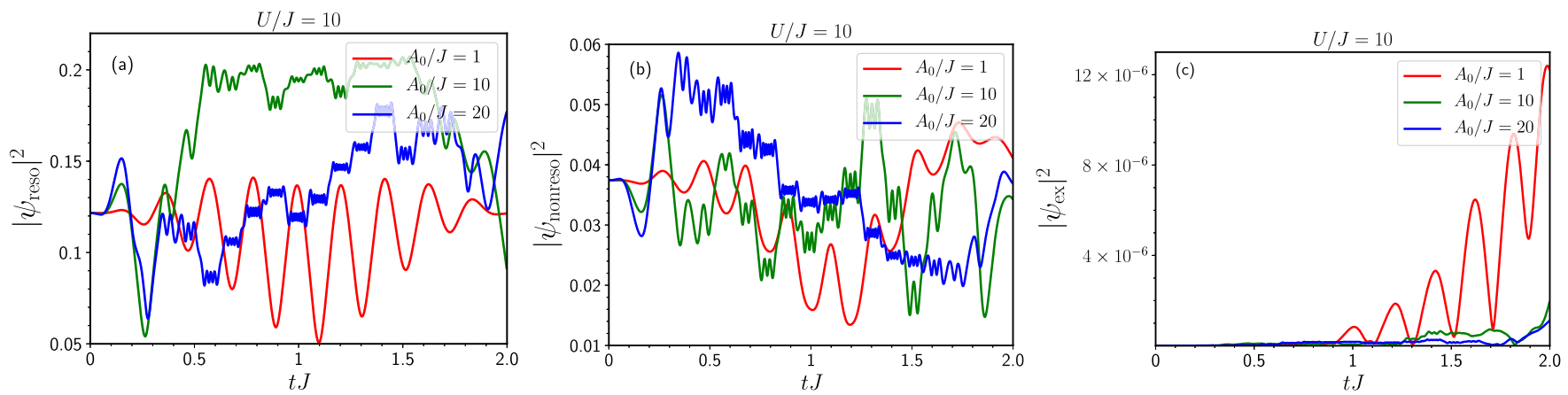

FIG. 5. Evolution of probability of finding (a) $|2011111\rangle$ (single-dipole state), (b) $|1012111\rangle$ (nonresonant states), and (c) $|5200000\rangle$ (highly excited state) states for varying strengths of $A_{0} / J$ in the Mott-insulating regime with $U / J=10$. Evidently, the evolution probability for the resonant dipole states [green curve in (a)] is found to be significant near $U / A_{0} \ll 1$, whereas for $U / A_{0} \gg 1$, the nonresonant state [blue curve in (b)] takes over the resonant one. The highly excited states are always suppressed [all curves in (c)] irrespective of the strength of light field.

$J$ leads to a resonant coupling between them. In addition to these, there are states where a quasiparticle-quasihole pair is not on nearest-neighbor sites. Such states are called nonresonant states. For further reading, the reader can consult Ref. [33].

Here we provide a representative example to identify resonant subspace for a system with $L=7$ sites and $N=7$ atoms. In this case, the neutral Mott state has one atom in each site, i.e., $|1111111\rangle$. At $E \sim U$, this state is coupled to the singledipole states |1201111 $\rangle,|1021111\rangle$, etc. These dipoles are

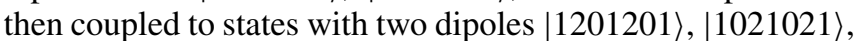
etc. These are further coupled to states with three dipoles, and so on. Note that these multiple-dipole states over the Mott states are part of the resonant family. As mentioned, the nonresonant states which are not made up of nearest dipoles can be expressed as $|1012111\rangle,|1101121\rangle$, etc. We note that the resonant and nonresonant states in the present scenario constitute a fraction of the total number of states. With this, we now aim to find the evolution of different resonant and nonresonant states in the presence of a synthetic vector potential with strength $A_{0}$, playing the role of $E$.

\section{Mechanism for HHG}

Having discussed the possible excitations in the Mott phase, we now investigate overlapping of resonant $\left(\left|\psi_{\text {reso }}\right|^{2}\right)$ and nonresonant states $\left(\left|\psi_{\text {nonreso }}\right|^{2}\right)$ with the evolved ground state $\Psi_{G}(t)$ of the system for different strength of the gauge potential. Indeed, this will help us in quantifying excitations responsible for HHG spectra as shown in Fig. 2. For illustration, we consider one resonant $|2011111\rangle$, one nonresonant $|1012111\rangle$, and one highly excited $|5200000\rangle$ state. Figure 5 shows the evolution of the probability of finding these states due to the application of the laser pulse. For weak strength of the potential $A_{0} \ll U$, the $\left|\psi_{\text {reso }}\right|^{2}$ oscillates follows the synthetic vector potential and the magnitude is very small. However, for $A_{0} \sim U$, we see enhanced $\left|\psi_{\text {reso }}\right|^{2}$, whereas $\left|\psi_{\text {nonreso }}\right|^{2}$ has an order of magnitude less contribution to the system. This is shown in Fig. 5(a). Thus the appearance of HHG can be attributed to the recombination of dipole states with the ground state at $A_{0} \sim U$. In contrast, the $\left|\psi_{\text {reso }}\right|^{2}$ reduces with increasing $A_{0}$, but the contribution for nonresonant states starts to dominate as evident from the blue line in Fig. 5(b). Thus, as expected, the HHG for stronger field is no longer due to the dipole states; rather the nonresonant states start to play a vital role. Apart from the resonant and nonresonant states, the excitation spectrum also consists of arbitrary highly excited states, for example, |5200000 $\rangle$. During the dynamics, the probability of contribution from these states is exceedingly low as is evident from Fig. 5(c) even when $A_{0} \geqslant U$. This trend is different from the behavior of resonant and nonresonant states in the spectrum. We find similar qualitative behavior for the other resonant, nonresonant, and highly excited states to the application of synthetic pulse with different strengths (not shown).

\section{Loschmidt echo}

To further analyze the dynamics towards the production of HHG, we turn our study to the evolution of ground-state population or Loschmidt echo, i.e., $\left|\left\langle\Psi_{G}(t=0) \mid \Psi(t)\right\rangle\right|^{2}$ for fixed $A_{0} / J>1$ and with varying $U / A_{0}$. It is evident from Fig. 6 that in the early stages of the laser pulse $t J \sim 0.4$, the ground state remains unaffected; that is, the population is close to unity. When $U=0$, that is, in the superfluid regime, any finite $A_{0}$ renders the single-particle wave function to be localized. The application of synthetic potential does not bring any substantial changes to the initial ground state. It is worth mentioning here that the effective amplitude $A_{0}(t)$ reaches maxima only at the half cycle $(t J=1)$ of the pulse. In the domain $U / A_{0}<1$, the nonresonant states along with the resonant dipole ones start to contribute. During the period $t J \sim 0.4$, the potential is too weak to excite these transitions; hence the population of the evolved state does not change. For $t J>0.4$, the amplitude of the vector potential becomes effectively strong enough to initiate modifying the population of the ground state. The initial state at $t=0$ gets deformed. As the vector potential becomes comparatively weak after the half cycle, the system tries to relax back to the ground state giving rise to a reentrant behavior. We now focus our attention on the regime when $U \sim A_{0}$. The dipole formation starts around the midcycle, and the initial Mott state gets deformed giving rise to a decrease in the population. Following the sinusoidal nature of the pulse, we find reentrant behavior of the ground state. The excited 


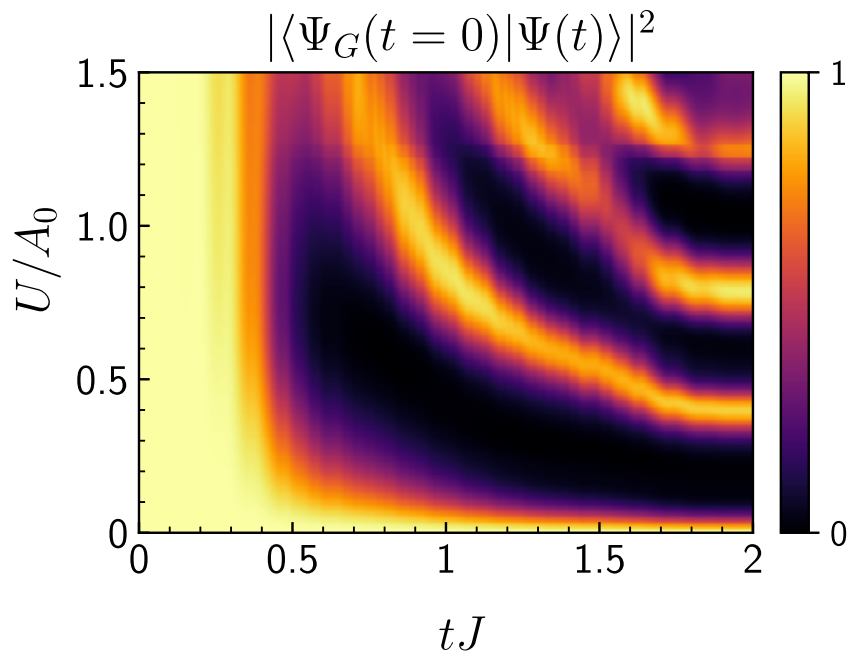

FIG. 6. False color coded image showing the variation in Loschmidt echo $\left|\left\langle\Psi_{G}(t=0) \mid \Psi(t)\right\rangle\right|^{2}$ with time for a fixed value of $A_{0} / J$ and varying $U / A_{0}$. In the limit of $U=0$, the initial ground state does not change appreciably, corroborating the localized nature of the ground state in the presence of light field [33]. In contrast, the Mott regime shows reentrant behavior due to recombination of excited states with the ground state in the presence of synthetic vector potential. This in turn leads to higher harmonics in the emitted spectra as elaborated in the main text.

state relaxes back and recombines with the ground state. It may be recalled here that $U \sim A_{0}$ is a necessary condition for the production of HHG. As $U / A_{0}>1$, the deformation of the initial Mott state gets plagued by weak vector potential. Hence, instead of the population going to zero, it remains as a finite quantity.

\section{CONCLUSIONS}

We have performed a detailed analysis to show that the Mott phase of the Bose-Hubbard model admits HHG production, whereas the SF phase does not. In particular, we have demonstrated here the mechanism behind the generation of higher harmonics using intense synthetic electric field in an interacting bosonic gas loaded in a one-dimensional optical lattice. We find that the strong synthetic time-dependent vector potential manifesting itself as the synthetic electric field can generate reasonably high harmonics in the insulating regime, while the superfluid regime is transparent to it. In the insulating regime, the order and number of harmonics increase with the variation in the strength of the synthetic vector potential translating to a linear cutoff law. This is attributed to the field-driven resonant and nonresonant excitations in the neutral Mott state and their subsequent recombination with the ground state. We have shown that if the repulsive interaction between two atoms $(U)$ is close to the strength of the potential $\left(A_{0}\right)$, the resonant quasiparticle-quasihole pairs on nearest-neighbor sites play a pivotal role in generating higher harmonics. However, in the strong-field limit $A_{0} \gg U$, the nonresonant states where quasiparticle-quasihole pairs are not located on the nearest-neighbor sites contribute to higher harmonics. Finally, we have indicated a possible experimental scope of the obtained results. In the future, investigation of HHG in square lattices would be a natural choice, where identifying and understanding dipole states in higher dimensions is itself a topic of fundamental importance. We believe that on one hand the creation of a near Mott insulator and on the other the experimental feasibility to study strong-field ionization in ultracold atoms [38] would make it feasible to study analogous HHG by bosons and its variants related to strong-field physics in the near future.

\section{ACKNOWLEDGMENTS}

A.R. acknowledges support from Provincia Autonoma di Trento. S.B. would like to thank G. Dixit, F. Evers, and S. Pujari for several discussions. S.B. acknowledges support from Department of Science and Technology, India, through Ramanujan Fellowship Grant No. SB/S2/RJN-128/2016 and through Early Career Award No. ECR/2018/000876, and MPG for funding through the Max Planck Partner Group at IITB. We thank the visitor program of Max Planck Institute for the Physics of Complex Systems, Dresden, for hospitality during the initial stages of the work. We also thank the anonymous referees for their thorough review and valuable comments, which contributed to improving the quality of the manuscript.

\section{APPENDIX A: CONVERGENCE CHECK OF HHG SPECTRUM FOR DIFFERENT TEMPORAL STEPS OF RUNGE-KUTTA ALGORITHM}

In this Appendix, we provide concrete evidence of numerical accuracy and convergence of the Runge-Kutta algorithm used in this present study. For different temporal steps in the Runge-Kutta algorithm, the HHG spectra in Fig. 7 are found to fall on top of each other, justifying the accuracy and convergence of the dynamical behavior.

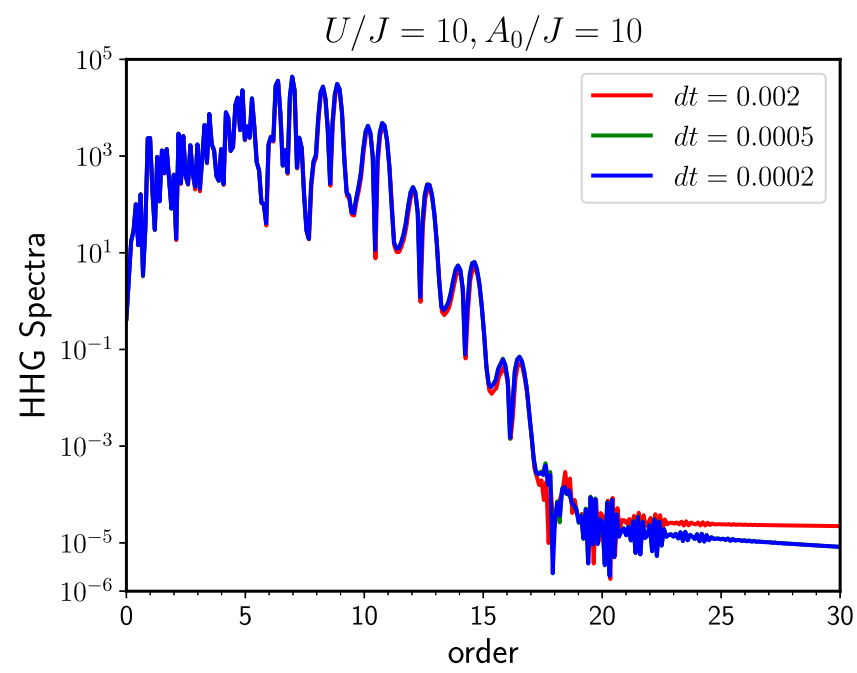

FIG. 7. Plots of HHG spectrum in the deep Mott-insulating regime for different temporal steps used in the Runge-Kutta algorithm. Evidently, the plots for different temporal steps match well with others, justifying the reliability and accuracy of the obtained dynamical behavior. 


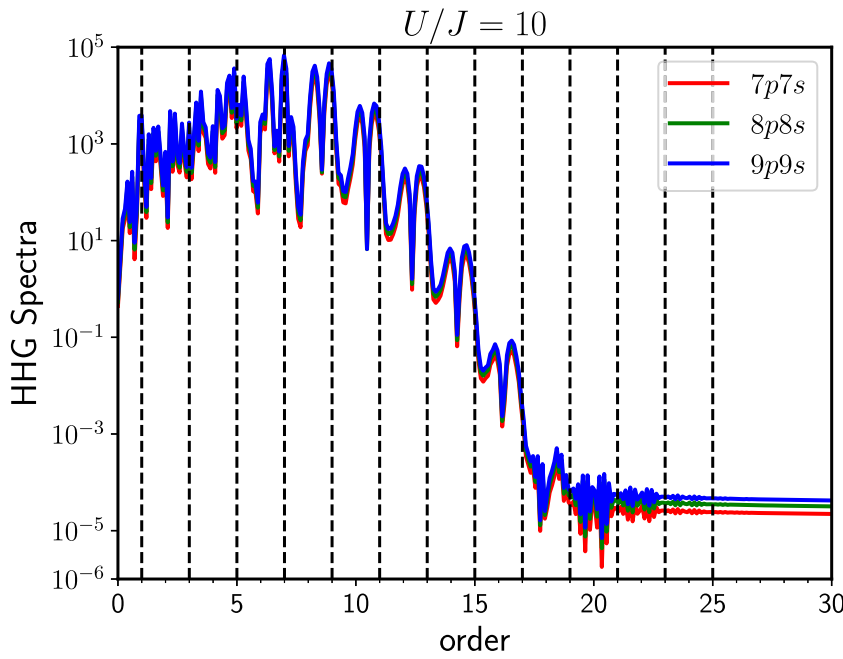

FIG. 8. Plots of HHG spectrum in the deep Mott-insulating regime for $A_{0} / J=10$ for different system sizes ranging from 7 particles $(p)$ in 7 sites $(s)$ to 9 particles $(p)$ in 9 sites $(s)$. Evidently, the plots for different configurations match well with others, justifying that our results are independent of system sizes.

\section{APPENDIX B: DEPENDENCE ON SYSTEM SIZE}

We provide here additional results to support that our calculations are devoid of any finite-size effects for the case $U / J=10$ and $A_{0} / J=10$ (see Fig. 8). As mentioned before, we used exact diagonalization for our computations and going beyond the configuration with 9 particles and 9 sites is computationally expensive. In view of this we would also like to point out that the generation of HHG in these systems is due to strong correlation and not mean field in nature; therefore to describe it one requires a full diagonalization of the system. This restricts the system sizes that we could access due to the exponential growth of the Hilbert space. Nevertheless, this is the state-of-the-art calculation and there also exist further works using exact diagonalization [39,50] (and complementing it with density matrix renormalization group [51]) in the bosonic model, where also convergence seems to be achieved within similar system sizes revealing qualitatively identical physics. All these give us confidence that our results will be valid in the thermodynamic limit.
[1] G. Vampa and T. Brabec, J. Phys. B 50, 083001 (2017).

[2] S. Takayoshi, Y. Murakami, and P. Werner, Phys. Rev. B 99, 184303 (2019).

[3] M. Lewenstein, P. Balcou, M. Y. Ivanov, A. L'Huillier, and P. B. Corkum, Phys. Rev. A 49, 2117 (1994).

[4] T. N. Ikeda and M. Sato, Phys. Rev. B 100, 214424 (2019).

[5] M. Ferray, A. L'Huillier, X. F. Li, L. A. Lompre, G. Mainfray, and C. Manus, J. Phys. B: At., Mol. Opt. Phys. 21, L31 (1988).

[6] A. L'Huillier, K. J. Schafer, and K. C. Kulander, Phys. Rev. Lett. 66, 2200 (1991).

[7] J. L. Krause, K. J. Schafer, and K. C. Kulander, Phys. Rev. A 45, 4998 (1992).

[8] J. J. Macklin, J. D. Kmetec, and C. L. Gordon, Phys. Rev. Lett. 70, 766 (1993).

[9] A. L'Huillier and P. Balcou, Phys. Rev. Lett. 70, 774 (1993).

[10] A. Baltuska, T. Udem, M. Uiberacker, M. Hentschel, E. Goulielmakis, C. Gohle, R. Holzwarth, V. S. Yakovlev, A. Scrinzi, T. W. Hänsch, and F. Krausz, Nature (London) 421 , 611 (2003).

[11] F. Krausz and M. Ivanov, Rev. Mod. Phys. 81, 163 (2009).

[12] S. Ghimire, A. D. DiChiara, E. Sistrunk, P. Agostini, L. F. DiMauro, and D. A. Reis, Nat. Phys. 7, 138 (2011).

[13] Y. S. You, Y. Yin, Y. Wu, A. Chew, X. Ren, F. Zhuang, S. Gholam-Mirzaei, M. Chini, Z. Chang, and S. Ghimire, Nat. Commun. 8, 724 (2017).

[14] M. Hohenleutner, F. Langer, M. K. O. Schubert, U. Huttner, S. Koch, M. Kira, and R. Huber, Nature (London) 523, 572 (2014).

[15] O. Schubert, M. Hohenleutner, F. Langer, B. Urbanek, C. Lange, U. Huttner, D. Golde, T. Meier, M. Kira, S. W. Koch, and R. Huber, Nat. Photonics 8, 119 (2014).

[16] T. T. Luu, M. Garg, S. Y. Kruchinin, A. Moulet, M. T. Hassan, and E. Goulielmakis, Nature (London) 521, 498 (2015).

[17] N. Yoshikawa, T. Tamaya, and K. Tanaka, Science 356, 736 (2017).
[18] S. G. G. Ndabashimiye, M. Wu, D. A. Browne, K. J. Schafer, M. B. Gaarde, and D. A. Reis, Nature (London) 534, 520 (2014).

[19] H. Liu, Y. Li, Y. S. You, S. Ghimire, T. F. Heinz, and D. A. Reis, Nat. Phys. 13, 262 (2014).

[20] T. N. Ikeda, K. Chinzei, and H. Tsunetsugu, Phys. Rev. A 98, 063426 (2018).

[21] S. Ghimire and D. A. Reis, Nat. Phys. 15, 10 (2019).

[22] K. Chinzei and T. N. Ikeda, Phys. Rev. Research 2, 013033 (2020).

[23] B. Cheng, N. Kanda, T. N. Ikeda, T. Matsuda, P. Xia, T. Schumann, S. Stemmer, J. Itatani, N. P. Armitage, and R. Matsunaga, Phys. Rev. Lett. 124, 117402 (2020).

[24] G. Vampa, T. J. Hammond, N. Thiré, B. E. Schmidt, F. Légaré, C. R. McDonald, T. Brabec, D. D. Klug, and P. B. Corkum, Phys. Rev. Lett. 115, 193603 (2015).

[25] M. Wu, S. Ghimire, D. A. Reis, K. J. Schafer, and M. B. Gaarde, Phys. Rev. A 91, 043839 (2015).

[26] H. Park, A. Camper, K. Kafka, B. Ma, Y. H. Lai, C. Blaga, P. Agostini, L. F. DiMauro, and E. Chowdhury, Opt. Lett. 42, 4020 (2017).

[27] M. Garg, H. Y. Kim, and E. Goulielmakis, Nat. Photonics 12, 291 (2018).

[28] R. E. F. Silva, I. V. Blinov, A. N. Rubtsov, O. Smirnova, and M. Ivanov, Nat. Photonics 12, 266 (2018).

[29] Y. Murakami, M. Eckstein, and P. Werner, Phys. Rev. Lett. 121, 057405 (2018).

[30] N. Tancogne-Dejean, M. A. Sentef, and A. Rubio, Phys. Rev. Lett. 121, 097402 (2018).

[31] Y. Murakami and P. Werner, Phys. Rev. B 98, 075102 (2018).

[32] S. Imai, A. Ono, and S. Ishihara, Phys. Rev. Lett. 124, 157404 (2020).

[33] S. Sachdev, K. Sengupta, and S. M. Girvin, Phys. Rev. B 66, 075128 (2002). 
[34] B. Yang, H. Sun, C.-J. Huang, H.-Y. Wang, Y. Deng, H.-N. Dai, Z.-S. Yuan, and J.-W. Pan, Science 369, 550 (2020).

[35] M. Greiner, O. Mandel, T. Esslinger, T. W. Hansch, and I. Bloch, Nature (London) 415, 39 (2002).

[36] Y.-J. Lin, R. L. Compton, K. Jiménez-García, W. D. Phillips, J. V. Porto, and I. B. Spielman, Nat. Phys. 7, 531 (2011).

[37] J. Dalibard, F. Gerbier, G. Juzeliūnas, and P. Öhberg, Rev. Mod. Phys. 83, 1523 (2011).

[38] P. Wessels, B. Ruff, T. Kroker, A. K. Kazansky, N. M. Kabachnik, K. Sengstock, M. Drescher, and J. Simonet, Commun. Phys. 1, 32 (2018).

[39] D. Jaksch, C. Bruder, J. I. Cirac, C. W. Gardiner, and P. Zoller, Phys. Rev. Lett. 81, 3108 (1998).

[40] I. Bloch, J. Dalibard, and W. Zwerger, Rev. Mod. Phys. 80, 885 (2008).

[41] M. P. A. Fisher, P. B. Weichman, G. Grinstein, and D. S. Fisher, Phys. Rev. B 40, 546 (1989).
[42] B. Capogrosso-Sansone, S. G. Söyler, N. Prokof'ev, and B. Svistunov, Phys. Rev. A 77, 015602 (2008).

[43] K. Sengupta and N. Dupuis, Phys. Rev. A 71, 033629 (2005).

[44] N. Goldman, G. Juzeliūnas, P. Öhberg, and I. B. Spielman, Rep. Prog. Phys. 77, 126401 (2014).

[45] C. Yu, S. Jiang, and R. Lu, Adv. Phys.: X 4, 1562982 (2019).

[46] G. Vampa, C. R. McDonald, G. Orlando, D. D. Klug, P. B. Corkum, and T. Brabec, Phys. Rev. Lett. 113, 073901 (2014).

[47] F. Gentile, A. Montorsi, and M. Roncaglia, Int. J. Quantum. Inform. 12, 1560014 (2014).

[48] B. M. Breid, D. Witthaut, and H. J. Korsch, New J. Phys. 9, 62 (2007).

[49] J. B. Krieger and G. J. Iafrate, Phys. Rev. B 33, 5494 (1986).

[50] J. M. Zhang and R. X. Dong, Eur. J. Phys. 31, 591 (2010).

[51] U. Schollwöck, Rev. Mod. Phys. 77, 259 (2005). 